\title{
Conversion énergie cinétique - énergie mécanique dans un moteur éolien
}

\author{
Gilles Feld, Emmanuel Hoang, Romain Dardevet, Éric Labouré \\ Adresse mél : gilles.feld@ens-cachan.fr, emmanuel.hoang@ens-cachan.fr, Romain.Dardevet@ac-nancy-metz.fr, \\ eric.laboure@satie.ens-cachan.fr, \\ Département EEA, ENS Cachan, 61 Avenue du Président Wilson, 94235 Cachan, Cedex
}

RÉSUMÉ : L’objectif de cet article est d'étudier la conversion d'énergie dans un moteur éolien à axe horizontal à partir d'une étude mécanique sur une hélice simplifiée. Pour des pales données et à vitesse de vent fixée, la caractéristique puissance mécanique en fonction de la vitesse angulaire de rotation est déterminée, ce qui permet de justifier l'intérêt d'un aérogénérateur à vitesse variable.

Mots clés : moteur éolien, portance, traînée, caractéristique de puissance d'une éolienne.

\section{INTRODUCTION}

La manipulation décrite dans ce document a été proposée lors d'un stage organisé pour des enseignants de physique, physique appliquée et génie électrique de l'académie de Créteil dans le cadre de la formation continue. Cette formation intitulée «Énergies nouvelles : de la physique à l'application » se décompose en deux parties. Une première partie théorique de douze heures où trois thèmes sont abordés : le photovoltaïque, l'éolien et la pile à combustible. Une deuxième partie expérimentale est composée de trois séances de travaux pratiques de six heures sur les thèmes précédemment cités. Le texte qui suit étudie la conversion énergie cinétique - énergie mécanique dans une éolienne. Il correspond à la première partie d'une étude expérimentale sur les moteurs éoliens. La deuxième aborde l'étude des aérogénérateurs à vitesse variable. Les enseignants ont appréciés cette étude qui a permis à partir de notions simples de physique de comprendre la conversion d'énergie dans un moteur éolien, de justifier la forme des pales et l'intérêt de la vitesse variable. Certains enseignants vont exploiter à très court terme cette formation dans leur enseignement. Nous allons proposer aux étudiants de master première année cette séance de travaux pratiques dans une unité d'enseignement traitant des énergies renouvelables.

\section{2 ÉTUDE D'UNE HELICE SIMPLIFIEE}

L'hélice simplifiée (cf. fig. 1) est constituée de trois pales planes. Elle est soumise à un vent de vitesse notée $\mathrm{V}$ (dirigée selon l'axe $\mathrm{Oz}$ ) qui est parallèle à l'axe de de rotation des pales, ce dernier tournant à la vitesse angulaire notée $\Omega$.

Nous isolons un tronçon élémentaire de pale situé à une distance $r$ du centre de rotation. On note 1 sa largeur, dr sa longueur et $\beta$ l'angle de calage (cf. fig. 2). Cet élément est soumis au vent réel de vitesse $\mathrm{V}$ et à un vent dû à la rotation de l'hélice de valeur $-\mathrm{V}_{\mathrm{x}}$, avec $\mathrm{V}_{\mathrm{x}}=\Omega . \mathrm{r}$. La somme vectorielle des effets de ces deux vents donne le vent apparent de vitesse notée $\mathrm{V}_{\mathrm{a}}$. L'angle d'incidence i est l'angle entre l'orientation du vent apparent et la pale. La figure 2 représente ces différentes grandeurs dans un repère lié au tronçon de pale. Ce vent apparent a pour module :

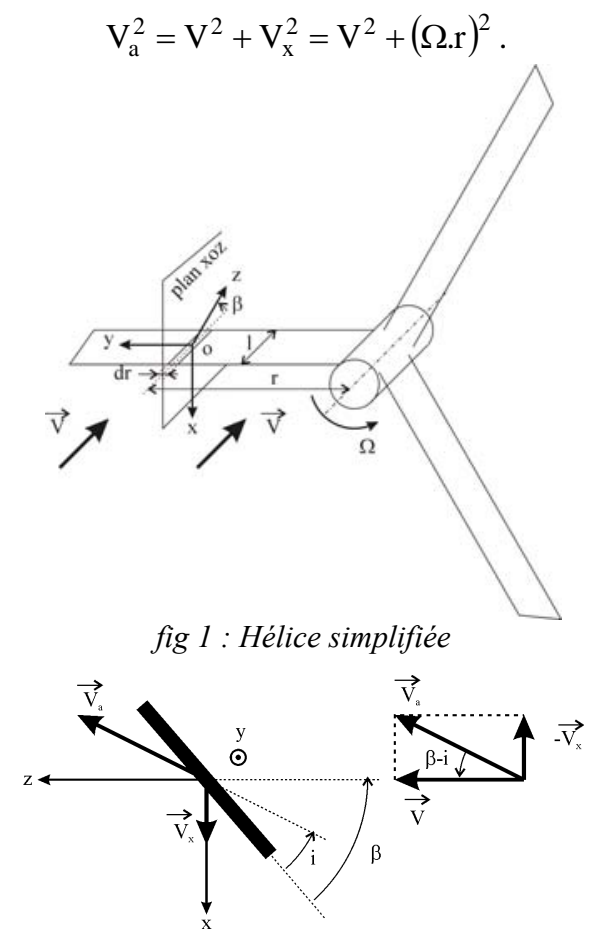

fig 2 : Vue de l'élément de pale dans le plan $x \mathrm{Oz}$

Pour un angle de calage donné, l'angle d'incidence est fonction de la vitesse du vent $(\mathrm{V})$ et de la vitesse due à la rotation $\left(\mathrm{V}_{\mathrm{x}}\right)$. En effet :

$$
\mathrm{i}=\beta-\operatorname{arctg}\left(\frac{\mathrm{r} . \Omega}{\mathrm{V}}\right)=\beta-\operatorname{arctg}(\lambda)
$$

où $\lambda$ est la vitesse spécifique du tronçon définie par :

$$
\lambda=\frac{\mathrm{r} . \Omega}{\mathrm{V}} .
$$

La figure 3 représente l'évolution de l'angle d'incidence en fonction de la vitesse spécifique pour un angle de calage donnée. Pour un angle de calage donné et à vitesse de vent donnée, l'angle d'incidence diminue lorsque la vitesse de rotation ou le rayon augmente. Pour un angle de calage et une vitesse de rotation donnés, l'angle d'incidence augmente lorsque la vitesse du vent augmente. 


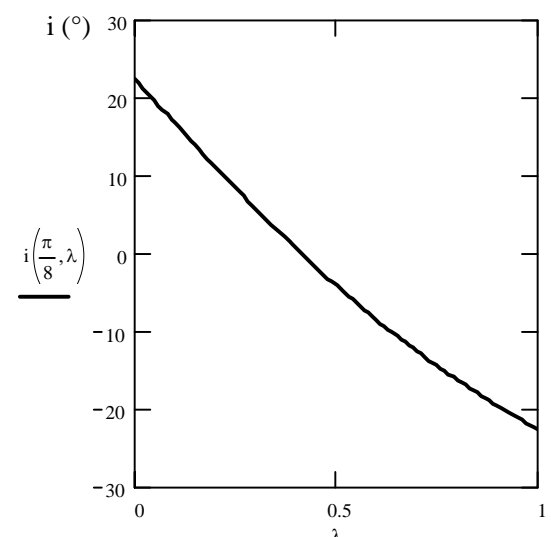

fig 3 : Évolution de l'angle d'incidence $i$ en fonction de la vitesse spécifique pour un angle de calage $\beta=\pi / 8$

En négligeant les effets des frottements, il s'exerce sur cet élément une force de pression (dR) perpendiculaire à la surface (analogue à la force de pénétration d'un véhicule dans l'air) dont le module s’écrit :

$$
\mathrm{dR}=\mathrm{k} \cdot \mathrm{V}_{\mathrm{a}}^{2} \cdot \mathrm{dS} \mathrm{S}^{\prime}
$$

où $\mathrm{dS}$ ’ représente la surface de la pale offerte au vent : $\mathrm{dS}^{\prime}=$ l.dr.sin(i),

d’où :

$$
\mathrm{dR}=\mathrm{k} \cdot \mathrm{V}_{\mathrm{a}}^{2} \cdot \mathrm{l} \cdot \sin (\mathrm{i}) \cdot \mathrm{dr} .
$$

Il est d'usage de décomposer cette force (dR) en :

- une force dans la direction du vent apparent (dT) appelée traînée,

- une force perpendiculaire à la direction du vent apparent (dP) appelée portance.

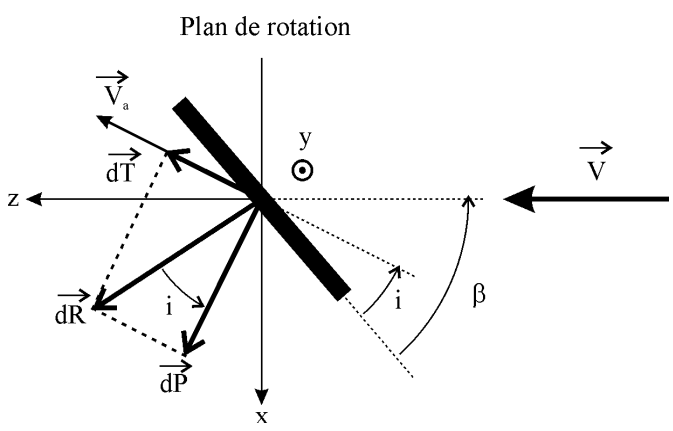

fig 4 : Force s'exerçant sur le tronçon élémentaire de la pale

Ainsi, on en déduit :

$$
\begin{gathered}
d T=d R \cdot \sin (i)=k \cdot V_{a}^{2} \cdot l \cdot \sin (i)^{2} \cdot d r \\
d P=d R \cdot \cos (i)=k \cdot V_{a}^{2} \cdot l \cdot \sin (i) \cos (i) \cdot d r .
\end{gathered}
$$

On définit [1] les coefficients de portance $C_{L}(L$ comme lift) et de traînée $C_{D}$ (D comme drag) de la façon suivante :

- force de traînée : dT $=\frac{1}{2} \rho \cdot C_{D} \cdot V_{a}^{2} \cdot l \cdot d r$,

- force de portance : dP $=\frac{1}{2} \rho \cdot C_{L} \cdot V_{a}^{2} \cdot l \cdot d r$,

où $\rho$ est la masse volumique de l'air $\left(\rho=1,225 \mathrm{~kg} / \mathrm{m}^{3}\right)$.
Dans notre cas d'école, les coefficients sont donc donnés par les expressions :

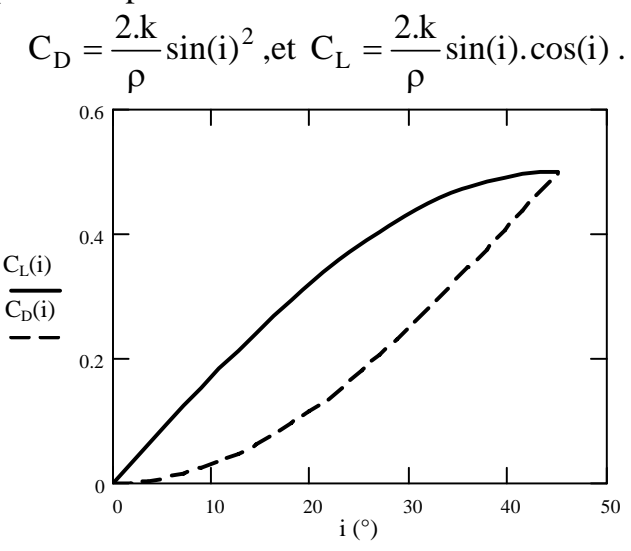

fig 5 : Évolution des coefficients de portance et de traînée en fonction de l'angle d'incidence en degré

Les deux forces élémentaires $\mathrm{dT}$ et $\mathrm{dP}$ vont engendrer une force motrice $\mathrm{dF}$ et un couple élémentaire :

$$
\mathrm{dC}=\mathrm{r} \cdot \mathrm{dF}=\mathrm{r}(\mathrm{dP} \cdot \cos (\beta-\mathrm{i})-\mathrm{dT} \cdot \sin (\beta-\mathrm{i}))
$$

On en déduit que la contribution de cet élément à la puissance mécanique est donnée par la relation :

$\mathrm{dP}_{\mathrm{m}}=\mathrm{dC} . \Omega$

$$
=\frac{1}{2} \rho \cdot r \cdot V_{a}^{2} \cdot \Omega \cdot l\left(C_{L}(i) \cdot \cos (\beta-i)-C_{D}(i) \cdot \sin (\beta-i)\right) d r
$$

où $V_{a}=f(V, \Omega, r)$ et $i=f(\beta, r, \Omega, V)$.

La figure 6 représente l'allure de la contribution à la puissance mécanique de ce tronçon pour un angle de calage et une vitesse de vent donnés.

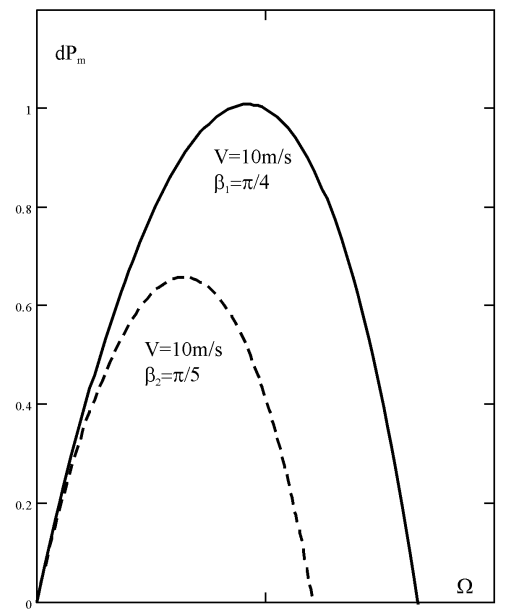

fig 6 : Évolution de la puissance en fonction de la vitesse angulaire de rotation pour une vitesse de vent donnée et deux angles de calage

À vitesse de vent donnée, la puissance passe par un maximum qui dépend de la vitesse du vent. Cette puissance s'annule pour un angle (i) d'incidence nul, soit encore pour : $\Omega=\frac{\mathrm{V}}{\mathrm{r}} \operatorname{tg}(\beta)$. 


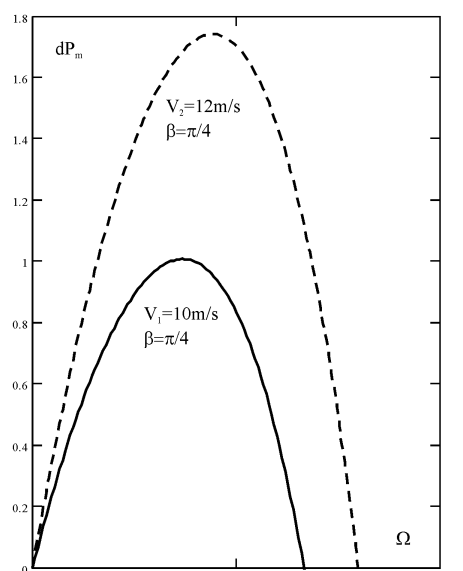

fig 7 : Évolution de la puissance en fonction de la vitesse angulaire de rotation pour un angle de calage donné et deux vitesses de vent

En conclusion de cette étude, nous pouvons noter les points suivants.

- Il faudrait pouvoir adapter la vitesse de rotation de l'hélice à la vitesse du vent pour optimiser la conversion d'énergie cinétique en énergie mécanique.

- La vitesse angulaire correspondant au maximum de puissance dépend de la vitesse du vent mais aussi de $r$ (position du tronçon de pale par rapport à l'axe de rotation). Il est donc nécessaire de vriller le profil de la pale ( $\beta$ est fonction de r) pour optimiser la conversion.

- Le calcul de la puissance totale (eq 1) se fait en intégrant la puissance élémentaire de 0 à $\mathrm{R}$, rayon de l’hélice :

$$
\mathrm{P}_{\mathrm{m}}=\int_{0}^{\mathrm{R}} \mathrm{dP}
$$

\section{EXEMPLE DE PALE REELLE [3]}

Prenons comme exemple une éolienne Vestas V80 de puissance $2 \mathrm{MW}$. La pale de cette éolienne se compose d'un profil aérodynamique NACA $63 \mathrm{XXX}$ entre le bout de la pale et le centre de la pale et d'un profil aérodynamique FFA W3 XXX entre le centre de la pale et le moyeu. La corde des profils le long de la pale varie entre 0,48 m (bout de la pale) et 3,52 m (base de la pale) sur une longueur de 35 mètres (cf. fig. 8). Le vrillage de la pale varie de $13^{\circ}$ (moyeu) à $0^{\circ}$ (bout de pale).

L'expression de la puissance élémentaire (eq 1) est toujours applicable dans le cas d'un profil réel. Dans ce cas on $\mathrm{a}: \beta=\beta_{0}+\mathrm{f}(\mathrm{r})$, où $\beta_{0}$ est l'angle de calage et $\mathrm{f}(\mathrm{r})$ l'angle de vrillage. La largeur des tronçons (l) à prendre en compte est la corde qui est fonction du rayon r. Par contre, les coefficients de portée et de traînée dépendent de la forme du profil, de la nature de l'écoulement et de l'angle d'incidence (i). Pour un profil donné, ces coefficients peuvent être déterminés par le calcul ou par mesures effectuées en soufflerie.

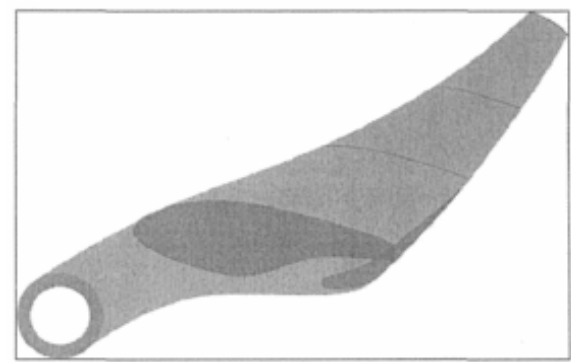

fig 8: Pale de l'éolienne Vestas V80 [3]

\section{4 ÉTUDE EXPERIMENTALE}

\subsection{Hélice étudiée}

Les caractéristiques de l'hélice sont :

- nombre de pales : 6 ,

- longueur d'une pale : 0,4 m,

- corde $: \mathrm{l}=$ constante $=22 \mathrm{~cm}$,

- angle de vrillage : 0 ,

- angle de calage : $\beta_{0}=\pi / 6$,

- profil, cf. photographie 1 ci dessous.

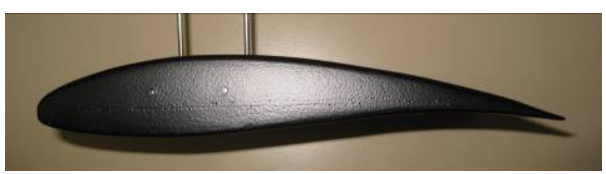

photo. 1 : Profil de la pale à étudier

\subsection{Mesure des coefficients de portance et de traînée}

La détermination de ces coefficients est effectuée à partir d'une étude en soufflerie (cf photo. 2) avec un tunnel d'essai muni d'une balance aérodynamique (cf photo. 3). La vitesse maximale du vent dans le tunnel est : $V_{\max }=8 \mathrm{~m} / \mathrm{s}$. La longueur de l'élément de pale est : $r=12 \mathrm{~cm}$. Pour différents angles d'incidence, nous déterminons les forces de traînée $(\mathrm{T})$ et de portance (P).

À partir de ces mesures effectuées pour la vitesse de vent maximum, nous en déduisons les évolutions des coefficients de portance et de traînée en fonction de l'angle d'incidence par les relations :

- coefficient de traînée : $\quad C_{D}=\frac{2 . T}{\rho . V^{2} \cdot \text { l.r }}$,

- coefficient de portance : $C_{L}=\frac{2 \cdot P}{\rho \cdot V^{2} \cdot . \cdot r}$.

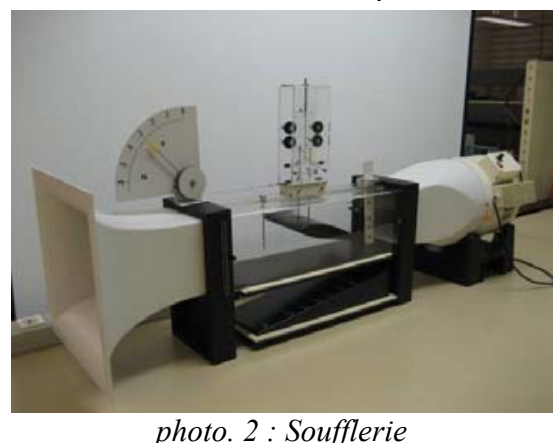

photo. 2 : Soufflerie 


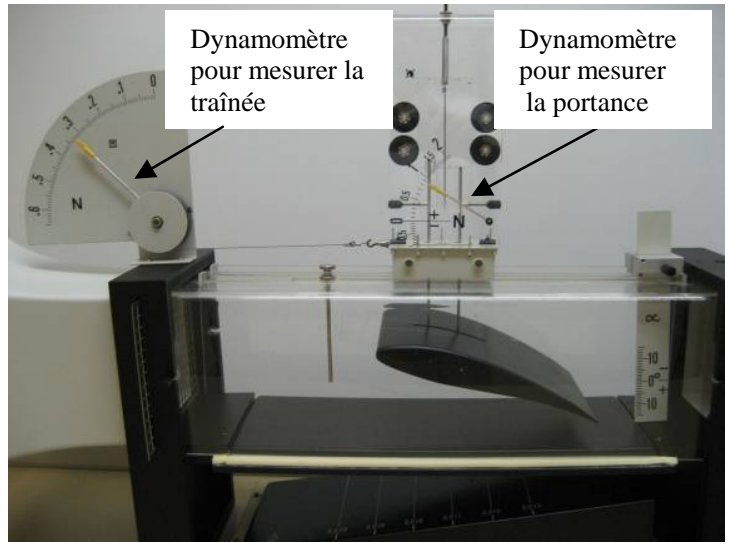

photo. 3 : Balance aérodynamique

Les courbes représentées sur les figures 10 et 11 montrent les évolutions des coefficients de traînée et de portance en fonction de l'angle d'incidence.

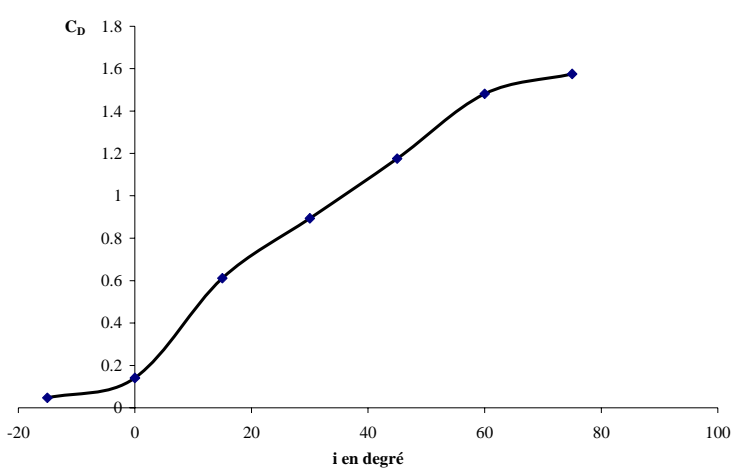

fig 9 : Relevé expérimental du coefficient de traînée en fonction de l'angle d'incidence

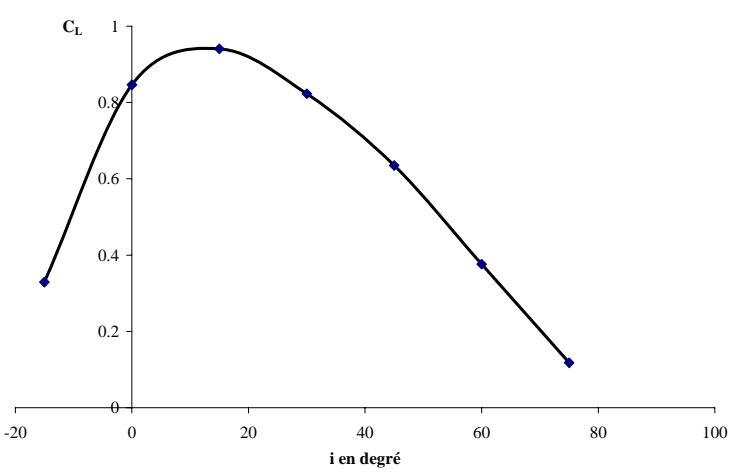

fig 10 : Relevé expérimental du coefficient de portance en fonction de l'angle d'incidence

\subsection{Détermination de la puissance}

La puissance est déterminée par intégration de l'expression donnée par l'équation 2. Pour effectuer cette intégration, nous avons décomposé chaque pale en 10 éléments de longueur $\Delta \mathrm{r}=4 \mathrm{~cm}$ et utilisé le logiciel Excel. De cette façon, nous pouvons vérifier que la contribution de chaque élément est différente et qu'il serait nécessaire de vriller la pale pour augmenter les performances. La puissance de chaque élément est :
$\Delta \mathrm{P}_{\mathrm{m}}=\frac{1}{2} \rho \cdot r \cdot \Omega \cdot \mathrm{V}_{\mathrm{a}}^{2} \cdot \mathrm{l}\left(\mathrm{C}_{\mathrm{L}}(\mathrm{i}) \cdot \cos \left(\beta_{0}-\mathrm{i}\right)-\mathrm{C}_{\mathrm{D}}(\mathrm{i}) \cdot \sin \left(\beta_{0}-\mathrm{i}\right)\right) \Delta \mathrm{r}$

avec $\mathrm{V}_{\mathrm{a}}^{2}=\mathrm{V}^{2}+(\Omega \cdot \mathrm{r})^{2}$ et $\mathrm{i}=\beta_{0}-\operatorname{arctg}\left(\frac{\mathrm{r} . \Omega}{\mathrm{V}}\right)$.

Prenons comme exemple une pale tournant à la vitesse angulaire $\Omega=30 \mathrm{rad} / \mathrm{s}$ dans un vent de $15 \mathrm{~m} / \mathrm{s}$. La puissance fournie par les différents tronçons est reportée dans le tableau ci-dessous. Dans ces conditions (angle de calage constant), l'extrémité de la pale a tendance à freiner l'hélice.

position du tronçon par rapport au centre en $\mathrm{cm}(\mathrm{r})$

\begin{tabular}{|c|c|c|c|c|c|c|c|c|c|}
\hline 2 & 6 & 10 & 14 & 18 & 22 & 26 & 30 & 34 & 38 \\
\hline
\end{tabular}

tab. 1 : Puissance fournie par les différents tronçons en fonction de leur distance au centre

La figure 11 donne la puissance mécanique fournie en fonction de la vitesse angulaire de rotation pour deux vitesses de vent.

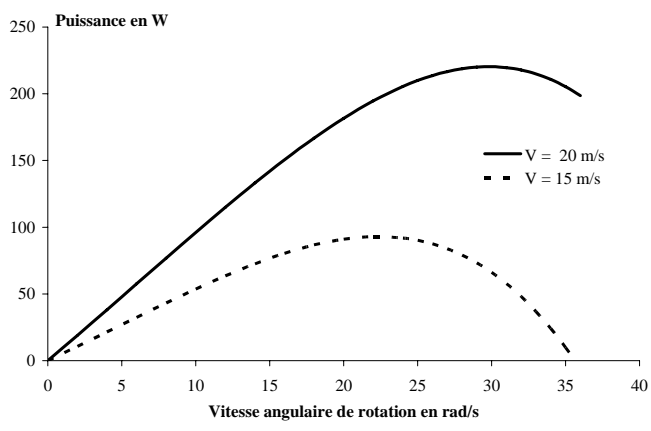

fig 11 : Évolution de la puissance en fonction de la vitesse de rotation pour deux vitesses de vent

\subsection{Conclusion}

L'étude précédente montre l'intérêt de pouvoir régler la vitesse de rotation des pales en fonction de la vitesse du vent. Pour une vitesse de vent de $15 \mathrm{~m} / \mathrm{s}$, l'hélice étudiée développe une puissance maximale d'environ $100 \mathrm{~W}$ pour une vitesse angulaire de $23 \mathrm{rad} / \mathrm{s}$ (fig. 11). Pour un vent de $20 \mathrm{~m} / \mathrm{s}$, la puissance passe à $230 \mathrm{~W}$ si les pales tournent à la vitesse de $30 \mathrm{rad} / \mathrm{s}$.

Pour qualifier l’efficacité de cette hélice, cette puissance peut être comparée à la puissance cinétique d'une colonne de vent de section $\mathrm{S}$ et animée d'une vitesse V (cf. fig. 12). Cette puissance [1] est donnée par :

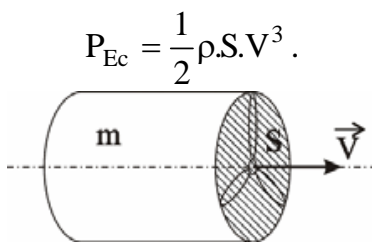

fig 12 :Colonne de vent 
Pour une vitesse de vent de $15 \mathrm{~m} / \mathrm{s}$, la puissance cinétique de la colonne de vent serait $\mathrm{P}_{\mathrm{Ec}} \approx 1000 \mathrm{~W}$. Pour récupérer intégralement cette puissance, il faudrait admettre que la vitesse de vent à la sortie de l'hélice est nulle. La vitesse étant non nulle en sortie, il en résulte que la veine fluide traversant le moteur éolien s'élargit (cf. fig. 13). La puissance maximum est donnée par la théorie de Betz [1] :

$$
\mathrm{P}_{\max \text { Betz }}=\frac{16}{27}\left(\frac{1}{2} \rho \cdot \mathrm{S} \cdot \mathrm{V}_{1}^{3}\right) .
$$

Pour une vitesse de vent de $15 \mathrm{~m} / \mathrm{s}, \mathrm{P}_{\text {max Betz }} \approx 600 \mathrm{~W}$,

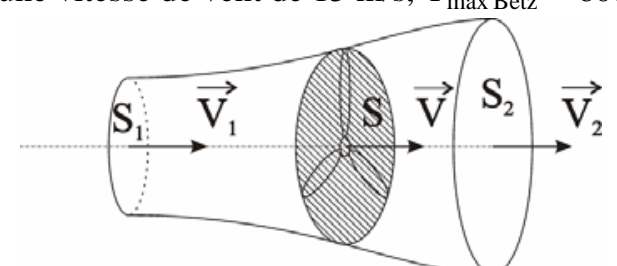

fig 13 : Veine de vent à la traversée de l'aéromoteur

Pour terminer cette comparaison, nous pouvons comparer la puissance délivrée par l'hélice étudiée à la puissance à la puissance annoncée par un constructeur d'éolienne de taille semblable. L'éolienne (photo. 4) de diamètre $91 \mathrm{~cm}$ développe une puissance de $300 \mathrm{~W}$ pour une vitesse de vent de $15 \mathrm{~m} / \mathrm{s}$.

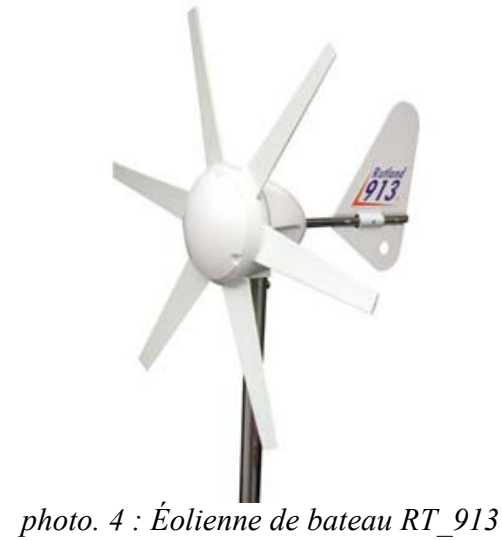

Le tableau suivant résume ces différent chiffres et l'on constate que les performances de l'hélice étudiée sont médiocres, elles pourraient être améliorées en vrillant les pales et en changeant de profil.

\begin{tabular}{|l|c|c|c|c|}
\hline $\begin{array}{l}\text { vitesse du } \\
\text { vent } 15 \mathrm{~m} / \mathrm{s}\end{array}$ & $\begin{array}{c}\text { hélice } \\
\text { étudiée }\end{array}$ & $\begin{array}{c}\text { colonne } \\
\text { de vent }\end{array}$ & $\begin{array}{c}\text { limite de } \\
\text { Betz }\end{array}$ & $\begin{array}{c}\text { éolienne } \\
\text { bateau }\end{array}$ \\
\hline puissance & $100 \mathrm{~W}$ & $1000 \mathrm{~W}$ & $600 \mathrm{~W}$ & $300 \mathrm{~W}$ \\
\hline
\end{tabular}

Pour compléter cette étude, nous pensons installer une centrale météorologique et étudier le potentiel éolien du site. Il serait donc possible à partir de l'histogramme des vitesses de vent de prédéterminer une hélice appropriée au site et de calculer l'énergie fournie par l'éolienne pendant une durée donné. Une étude sur les fluctuations de puissance pourrait être menée et permettrait d'aborder la problématique tech- nique économique et environnementale du de stockage de l'énergie.

\section{CONCLUSION}

Certes, l'hélice étudiée n'est pas très performante mais les objectifs sont atteints, nous avons montré l'intérêt de la vitesse variable, la nécessité de vriller les pales et surtout nous avons analysé le principe de conversion d'énergie dans un moteur éolien avec des outils simples

Cette séance de travaux pratiques est suivie d'une étude de la conversion mécanique-électrique dans les éoliennes et permet d'aborder la production d'énergie à partir de génératrices asynchrones à induction, de génératrices asynchrones à double alimentation et de génératrices synchrones.

Des études semblables allant de la physique à l'application ont été menées sur les deux autres thèmes : pile à combustible et photovoltaïque. Pour chaque séance, nous avons constaté un intérêt des stagiaires. Il est effectivement très satisfaisant de partir des principes physiques et ensuite d'appliquer ces principes sur des sujets à la mode.

\section{Bibliographie}

[1] D. Le Gouriéres, "Les Éoliennes", Éditions du Moulin Cadiou.

[2] M. Budinger, D. Leray, Y. Debleser, "Éoliennes et Vitesse Variable", Revue 3EI, $n^{\circ} 20$, mars 2000.

[3] F. Renaud, "Calcul des coefficients aérodynamiques d'un profil d'aile NACA", Mémoire, université du Québec. 